\title{
Einfluss zentralnervös dämpfender Arzneimittel auf Stürze mit Verletzungsfolgen bei Menschen mit Demenz in Pflegeheimen
}

\author{
Impact of Sedating Drugs on Falls Resulting Injuries Among People \\ with Dementia in a Nursing Home Setting
}

\section{(3) $\odot \Theta$}

\author{
Autoren \\ Theresa Lippert ${ }^{1}$, Renke Maas ${ }^{2}$, Martin F. Fromm², Katharina Luttenberger ${ }^{1}$, Peter Kolominsky-Rabas ${ }^{3}$, \\ Anna Pendergrass' ${ }^{1}$, Elmar Gräßel ${ }^{1}$
}

Institute

1 Zentrum für Medizinische Versorgungsforschung, Psychiatrische und Psychotherapeutische Klinik, Universitätsklinikum Erlangen, Friedrich-Alexander-Universität Erlangen-Nürnberg (FAU)

2 Institut für Experimentelle und Klinische Pharmakologie und Toxikologie, Lehrstuhl für Klinische Pharmakologie und Klinische Toxikologie, Friedrich-Alexander-Universität Erlangen-Nürnberg, Erlangen

3 Interdisziplinäres Zentrum für Health Technology Assessment (HTA) und Public Health (IZPH), FriedrichAlexander-Universität Erlangen-Nürnberg (FAU), Erlangen

Schlüsselwörter

Demenz, Sturz, Arzneimittel, Dämpfung

Key words

Dementia, Fall, Drugs, Sedation

Bibliografie

DOI https://doi.org/10.1055/a-1071-7911

Gesundheitswesen 2020; 82: 14-22

(c) Georg Thieme Verlag KG Stuttgart · New York

ISSN 0941-3790

Korrespondenzadresse

Theresa Lippert

Universitätsklinikum Erlangen

Psychiatrische und Psychotherapeutische Klinik

Zentrum für Medizinische Versorgungsforschung

Schwabachanlage 6

91054 Erlangen

theresa_lippert@web.de

Die Ratingliste finden Sie online unter http://dx.doi. org/10.1055/a-1071-7911.

\section{ZUSAMMENFASSUNG}

Ziel der Studie Ziele der Studie waren einerseits die Erstellung einer Skala zur Erfassung zentral dämpfender bzw. aktivierender Wirkungen von Arzneimitteln sowie andererseits die Analyse der Fragestellung, ob dieser Summenwert bei Demenzerkrankten einen signifikanten Zusammenhang mit behandlungsbedürftigen Sturzereignissen aufweist. Explorativ wurde zusätzlich das Verschreibungsverhalten im Pflegeheim hinsichtlich PRISCUS-Liste, Anticholinergic Cognitive Burden List (ACB-Liste) und Psychopharmaka untersucht.

Methodik Es wurden Daten einer randomisiert-kontrollierten Studie zur Untersuchung der Wirksamkeit der nicht-medikamentösen multimodalen Gruppentherapie MAKS ${ }^{\circledR}$ bei 139 Personen mit degenerativer Demenz in 5 Pflegeheimen evaluiert. Alle zum Zeitpunkt Studienbeginn verschriebenen Arzneimittel wurden hinsichtlich ihrer dämpfenden bzw. aktivierenden Wirkung auf einer 5-stufigen Skala von 2 klinischpharmakologischen Experten bewertet: Von stark aktivierend $($ Wert +2$)$ bis stark dämpfend (Wert -2$)$. Die so ermittelten Werte aller verschriebenen Arzneimittel wurden für jede Person zu einem „Dämpfungsscore“ aufaddiert. Der Zusammenhang zwischen „Dämpfungsscore“ und Sturzereignissen mit Verletzungsfolge in einem Zeitraum von 12 Monaten wurde mithilfe der binär-logistischen Regressionsanalyse untersucht. Ergebnisse Knapp 30\% aller Personen erhielten Arzneistoffe der PRISCUS-Liste, 50 \% bekamen einen Wirkstoff der ACBListe, $55 \%$ erhielten Psychopharmaka und $66 \%$ wurden mit mindestens fünf Arzneimitteln behandelt. $62 \%$ der Demenzerkrankten erhielten zentralnervös dämpfende Wirkstoffe. Im Beobachtungszeitraum kam es bei 36 der 139 Bewohnerinnen und Bewohner zu Sturzereignissen mit Verletzungsfolge. Der „Dämpfungsscore“ steht, multivariat analysiert, in signifikantem Zusammenhang ( $p=0,045)$ mit Sturzereignissen mit Verletzungsfolge. Eine stärkere zentralnervöse Dämpfung führte zu häufigeren Sturzereignissen.

Schlussfolgerung Der „Dämpfungsscore“ ist ein geeignetes Maß, um das Ausmaß der zentralnervösen Dämpfung zu beschreiben. Aufgrund des signifikanten Einflusses der Dämpfung auf Sturzereignisse mit Verletzungsfolge sollte bei der Medikation von 
Demenzerkrankten stärker darauf geachtet werden, möglichst wenig zentral dämpfende Arzneimittel einzusetzen, um das Risiko für relevante unerwünschte Wirkungen zu reduzieren.

\section{ABSTRACT}

Aim The aims of this study were to create a scale for measuring the sedating and activating effects of drugs and to analyse if the total value of this scale correlates significantly with falls requiring medical treatment in dementia patients. Furthermore, prescription of drugs in nursing homes included in the PRISCUS-List, Anticholinergic Cognitive Burden List (ACB-List) and usage of psychotropic drugs were investigated.

Method This is a data analysis of a randomized controlled trial which tested the effects of a non-pharmacological multimodal activation therapy $\left(\right.$ MAKS $\left.^{\circledR}\right)$ in 139 patients with degenerative dementia in 5 nursing homes. At the beginning of the study, all prescribed drugs were rated on a five-tier scale by 2 pharmacologists based on the drugs' sedating or activating effects. The scale ranged from severely activating $(+2)$ to severely sedating $(-2)$. The "central nervous system (CNS) de- pressant score" of each patient was calculated by summing up the scale value of all the medications they were taking. The correlation between CNS-depressant score and falls resulting in injuries within an observation period of 12 months was investigated by binary logistic regression analysis.

Results Nearly $30 \%$ of the nursing home residents received drugs listed in the PRISCUS-list, $50 \%$ received drugs on the ACBList, $55 \%$ took psychotropic drugs and $66 \%$ received at least 5 drugs. Sedating drugs were prescribed to $62 \%$ of patients. During the observation period, 36 out of 139 nursing home residents suffered falls and medical treatment was necessary. In multivariate analysis, the CNS-depressant score was associated significantly ( $p=0.045)$ with falls with resulting injuries. Increased sedation resulted in a higher number of fall incidents. Conclusions The CNS-depressant score is a useful tool to describe the degree of sedation. Due to the significant association between sedation and falls resulting in injuries, the sedating medication of people suffering from dementia should be minimised as much as possible to reduce the risk of undesirable side effects.

\section{Einleitung}

Neben der großen epidemiologischen Relevanz der Demenzerkrankungen für Deutschland stiegen die Krankheitskosten für Demenzerkrankte von 2002 bis 2015 von etwa 8,0 auf rund 15,1 Milliarden Euro [1, 2]. In diesem Zusammenhang haben Stürze mit ärztlich behandlungsbedürftigen Folgen eine besonders große Bedeutung, da bei Demenzerkrankten ein deutlich erhöhtes Sturzrisiko vorliegt und Sturzereignisse oft mit schwerwiegenden gesundheitlichen Folgen einhergehen $[3,4]$. Ein vermehrtes Auftreten von Stürzen wird in der Literatur häufig mit der Einnahme von Arzneimitteln aus bestimmten Medikamentengruppen in Verbindung gebracht. Einige Studien konnten erhöhte Sturzraten bei Pflegeheimbewohnerinnen und -bewohnern bei Einnahme von Psychopharmaka [5], sowie bei den Untergruppen Antidepressiva, Antipsychotika oder Sedativa feststellen [6-8]. Die FINALEX-Studie aus dem Jahr 2017 [9] belegt für Demenzerkrankte höhere Sturzraten bei Polypharmazie sowie bei Einnahme von Psychopharmaka oder anticholinerg wirkenden Arzneimitteln. Beispielsweise bei Polypharmazie steigt mit zunehmender Anzahl an Arzneimitteln die Wahrscheinlichkeit, dass darunter auch Arzneimittel sind, die das Sturzrisiko signifikant erhöhen. Die Vorhersage ist jedoch relativ ungenau, da unter den eingenommenen Arzneimitteln auch solche sein könnten, die nicht im Zusammenhang mit einem erhöhten Sturzrisiko stehen. Aus diesem Grund sind spezifischere pharmakologische Parameter zur Prädiktion von Stürzen wünschenswert. Vor allem, wenn die Sturzfolgen nicht nur gesundheitsökonomisch relevant sind, wie bei ärztlich behandlungsbedürftigen Sturzfolgen, sondern auch für die Betroffenen mit mehr Leid und Komplikationen verbunden sind, wie bei einem Knochenbruch, v. a. beim Oberschenkelhalsbruch im höheren Lebensalter, der mit einer nicht unerheblichen Mortalität assoziiert ist [10].
In der vorliegenden Arbeit wird der Arzneimitteleinsatz bei Demenzerkrankten hinsichtlich der demenzrelevanten Kriterien PRISCUS-Liste, ACB-Liste (Anticholinergic Cognitive Burden List), der Einnahme von Psychopharmaka sowie bezüglich Polypharmazie untersucht. Außerdem stellen wir die Entwicklung eines Summenwertes vor, der den einzelnen Wirkstoffen verschiedener Arzneimittelklassen einen bestimmten zentralnervösen, psychomotorischen Dämpfungsgrad zuordnet. Der aufaddierte Summenwert wird als „Dämpfungsscore“ bezeichnet. In der vorliegenden Analyse soll die klinische Bedeutung der zentralnervösen psychomotorischen Dämpfung im Zusammenhang mit Stürzen mit ärztlich behandlungsbedürftiger Verletzungsfolge untersucht werden.

\section{Methodik}

\section{Design}

In der vorliegenden Arbeit wurden die Daten einer multizentrischen, randomisiert-kontrollierten Verlaufsstudie analysiert, die den Einfluss der nichtmedikamentösen Gruppentherapie MAKS ${ }^{\circledR}$ auf Patientinnen und Patienten mit degenerativer Demenz untersuchte [11-13]. Die MAKS ${ }^{\circledR}$ Intervention beinhaltet vier therapeutische Komponenten: Training motorischer $(\mathrm{M})$ und alltagspraktischer (A) Fähigkeiten, kognitives Training (K) in der Gruppe sowie eine sozial-kommunikative (S) Komponente. Es handelt sich hierbei um eine standardisierte psychosoziale Intervention, die an sechs Tagen pro Woche 2 Stunden täglich von geschulten Therapeutinnen und Therapeuten in den Pflegeheimen durchgeführt wurde. Die Studie wurde in 5 Pflegeheimen in Bayern an insgesamt 139 Studienteilnehmerinnen und -teilnehmern durchgeführt. Es wurden die Eingangsdaten aller eingeschlossenen Personen sowie 
die Daten zur Sturzhäufigkeit (Outcome) während der folgenden 12 Monate verwendet. Die verfügbaren Informationen zum Outcome wurden für die Analyse bis zum Dropout berücksichtigt. Einschlusskriterien waren das Vorliegen einer ärztlich bestätigten primär degenerativen Demenz nach ICD-10 (F00, F03 oder G30), ein Ergebnis von weniger als 24 Punkten im Mini-Mental-Status-Test (MMST) [14], sowie das schriftliche Einverständnis der teilnehmenden Pflegeheimbewohnerinnen und -bewohner bzw. der gesetzlichen Betreuerinnen und Betreuer. Ausschlusskriterien waren das Vorliegen einer vaskulären (F01) oder sekundären Demenz (F02) sowie eine andere neurologisch-psychiatrische Erkrankung (Sucht, schwere Depression, Psychose), Pflegestufe 3, Bettlägerigkeit, Blindheit oder Taubheit. 141 Personen erfüllten zu Studienbeginn die Einschlusskriterien, 2 von ihnen mussten aufgrund eines nachträglich festgestellten Ausschlusskriteriums wieder aus dem Pool der geeigneten Personen entfernt werden. Die verbleibenden 139 Personen waren Grundlage dieser Analyse ( $\mathbf{A b b}$. 1). Die Ethikkommission der Medizinischen Fakultät der Universität ErlangenNürnberg hat das Studienprotokoll geprüft und zustimmend bewertet (Registriernummer 3232).

\section{Zielgröße}

Sturzereignisse mit Verletzungsfolge

Während der 12-monatigen Interventionsphase wurden Sturzereignisse mit Verletzungsfolge, die einer ärztlichen Behandlung bedurften - ambulant oder stationär - von den beteiligten Pflegeheimen standardisiert im Rahmen der Serious Adverse Event (SAE)-Erhebung dokumentiert. Hierzu erfolgte eine Schulung zur Datenerhebung. Zur Qualitätssicherung wurde stichprobenartig die Dokumentation von Angestellten der Studienzentrale überprüft.

\section{Pharmakologische Parameter}

Medikamentenanzahl

Durch die Erfassung aller verordneten Arzneimittel, die in der Dokumentation des Pflegeheims enthalten waren, wurde der Summenwert der verordneten Arzneimittel gebildet $(0,1,2,3, \ldots \geq 10)$. Es wurden nur Arzneimittel mit primär systemischer Wirkung und dauerhafter Verordnung eingeschlossen. Das heißt, alle Arzneimittel, die lokal angewendet wurden, sowie Bedarfsmedikation waren ausgeschlossen. Zu jedem Arzneimittel wurde die zugehörige 5-stellige Kennziffer der Roten Liste erfasst. Die Anzahl unterschiedlicher Kennziffern der Roten Liste jedes Heimbewohners ergab die individuelle Medikamentenanzahl. Als Polypharmazie wurde die Einnahme von 5 oder mehr Wirkstoffen definiert [15].

\section{PRISCUS-Liste}

Die PRISCUS-Liste [16] führt Arzneimittel auf, die beim Einsatz bei älteren Menschen ein erhöhtes Risiko für Nebenwirkungen zeigen. Sie enthält 83 Arzneistoffe, die nach einer strukturierten Expertenbefragung erarbeitet wurden. Bei Verschreibung eines dieser Arzneistoffe beträgt der PRISCUS-Score 1. Bei Einnahme von 2 Arzneistoffen der Liste ist der PRISCUS-Score 2, usw.

\section{ACB-Liste}

Die ACB-Liste (Anticholinergic Cognitive Burden List) klassifiziert anticholinerge Arzneimittel entsprechend ihrer Assoziation mit an- ticholinergen Wirkungen in 3 Gruppen: „possible anticholinergics“ - Score 1, „definite anticholinergics“ - Score 2 und „definitive anticholinergics associated with delirium “ - Score 3 . Für die Analyse wurde ein ACB-Score, d. h. ein Summenwert für jede Person ermittelt, der die einzelnen ACB-Werte der verordneten Arzneimittel aufsummiert. In dieser Arbeit wurde eine modifizierte Form der ACB-Liste von 2012 [17] nach Pfistermeister et al. [18] verwendet. Gemäß der ACB-Liste haben u. a. Haloperidol und Venlafaxin einen Score von 1, u. a. Carbamazepin und Pimozid einen Score von 2 sowie u. a. Amitryptilin und Doxepin einen Score von 3. Gemäß Pfistermeister et al. [18] wurde Trospium wegen seiner sehr limitierten ZNS-Gängigkeit von der ACB-Liste gestrichen. Zudem wurden Biperiden, Metixen und Maprotilin als „definite anticholinergics“ mit einem Score von 3 aufgenommen.

\section{Psychopharmaka}

Weiterhin wurde untersucht, wie viele der eingeschlossenen Personen Psychopharmaka erhielten. Laut der Roten Liste 2018 zählen Antidepressiva, Antipsychotika, Psychostimulanzien zur ADHSTherapie, Tranquillanzien und Anxiolytika sowie Lithium zur Hauptgruppe der Psychopharmaka. Als Psychopharmaka in dieser Arbeit wurden alle genannten Wirkstoffklassen sowie Pregabalin gezählt, da für diesen Wirkstoff ein Wirksamkeitsnachweis in der Therapie von generalisierten Angststörungen vorliegt [19].

\section{Dämpfungsscore}

Zur Ermittlung eines summativen Wertes für die zentralnervös dämpfende Wirkung von Arzneimitteln, als „Dämpfungsscore“ bezeichnet, wurden alle verordneten Arzneimittel der Patientendokumentation erfasst (zum Zeitpunkt Studienbeginn). Als Einschlusskriterien galten die dauerhafte Einnahme und eine systemische Wirkung. Durch einen voneinander unabhängigen Ratingprozess zweier pharmakologischer Experten (Koautoren) wurden alle verordneten Wirkstoffe auf Basis der aktuellen wissenschaftlichen Literatur und der Fachinformationen bezüglich ihrer zentralnervös aktivierenden beziehungsweise dämpfenden Wirkung bewertet. Hierbei wurde eine 5-stufige Skala von - 2 (stark sedierend), - 1 (schwach sedierend), 0 (weder sedierend noch aktivierend), 1 (schwach aktivierend) bis 2 (stark aktivierend) zugrunde gelegt. Die Liste aller bewerteten Arzneimittel ist der Publikation beigefügt (siehe Anlage: „Ratingliste“). Der Dämpfungsgrad der einzelnen Wirkstoffe eines Patienten wurde zu einem „Dämpfungsscore“ aufaddiert. Eine Dämpfung von -1 bis -2 wurde als mäßige Dämpfung definiert, eine Dämpfung von -3 bis -6 hingegen als starke Dämpfung. Ein Dämpfungsscore von $\leq-3$ kann nur durch eine Kombination mehrerer dämpfender Arzneimittel erreicht werden.

\section{Weitere Variablen}

\section{Mini-Mental-Status-Test}

Vor Einschluss in die Studie wurde bei jeder Bewohnerin und jedem Bewohner der Mini-Mental-Status-Test (MMST) durchgeführt [14]. Der MMST ist der international am häufigsten verwendete Screeningtest für Demenz. Er besitzt eine Spannweite von 0 bis 30 Punkten. Je höher der Wert, desto besser ist die gezeigte kognitive Leistung. 


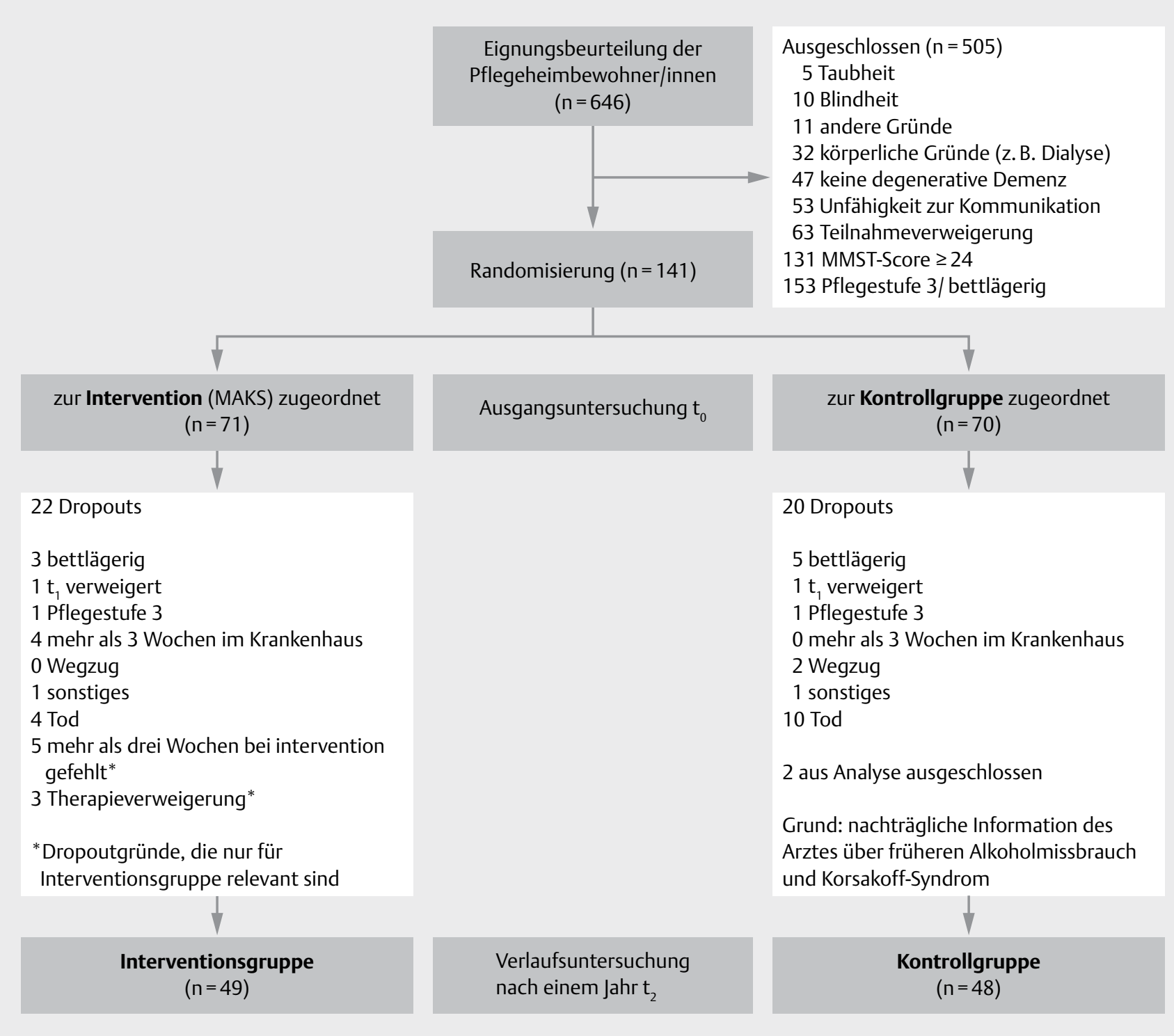

- Abb. 1 CONSORT-Flussdiagramm des Studiendesigns der MAKS-Studie (nach [12]).

Nurses' Observation Scale for Geriatric Patients (NOSGER) Ebenfalls wurde zu Beginn der Studie für jede eingeschlossene Person der NOSGER-Summenwert [20] ermittelt. Er beurteilt die geriatrische Gesamtsymptomatik. Die Fremdbeurteilungsskala umfasst 30 Items, von denen je 5 einer der folgenden 6 Dimensionen zugeordnet sind: Gedächtnis, IADL (Instrumentelle Aktivitäten des täglichen Lebens), ADL (basale Aktivitäten des täglichen Lebens wie Urin- / Stuhlgangkontrolle, eigenständige Körperpflege oder gepflegtes und sauberes Erscheinungsbild), Stimmung, soziales Verhalten sowie störendes Verhalten. Je höher die erreichte Punktzahl, desto mehr geriatrische Auffälligkeiten zeigt der Betroffene. Die Spannweite reicht je Dimension von 5 Punkten (keine Störung) bis 25 Punkte (maximal mögliche Störung). Somit ergibt sich eine mögliche Spanne des Summenwertes von 30 Punkten (keine Störung) bis 150 Punkten (maximale Störung).

\section{Pflegestufe}

Die Pflegestufe beschreibt die Einteilung der Pflegebedürftigkeit nach SGB XI (Pflegeversicherungsgesetz) vor Einführung der Pflegegrade. Das Ausmaß der Pflegebedürftigkeit wird wie folgt eingeteilt: Keine Pflegebedürftigkeit (keine Pflegestufe), mäßige Pflegebedürftigkeit (Pflegestufe 1), hohe Pflegebedürftigkeit (Pflegestufe 2) sowie sehr hohe Pflegebedürftigkeit (Pflegestufe 3). Bei allen Teilnehmern der Studie wurde zum Studienbeginn die Pflegestufe erfasst. Als Ausschlusskriterium wurde Pflegestufe 3 festgesetzt. In der Sensitivitätsanalyse wurde die Pflegestufe als dichotome Variable eingeschlossen (keine Pflegestufe oder Pflegestufe $1=0$, Pflegestufe 2 =1).

\section{Statistische Analyse}

In der deskriptiven Analyse wurde die Häufigkeit von Sturzereignissen mit Verletzungsfolge, das Verschreibungsverhalten bezüglich Wirkstoffen der PRISCUS- sowie der ACB-Liste, die Verschreibung 
- Tab. 1 Charakteristika der Stichprobe $(n=139)$.

\begin{tabular}{|c|c|c|c|c|}
\hline \multirow[t]{2}{*}{ Variable } & \multirow[t]{2}{*}{ Kohorte $^{b}(n=139)$} & \multicolumn{3}{|c|}{ Sturz mit Verletzungsfolge ${ }^{a}$} \\
\hline & & $\mathrm{Ja}^{\mathrm{b}}(\mathrm{n}=36)$ & Nein $^{b}(n=103)$ & $p$-Wertc \\
\hline Alter, in Jahren & $84,7(4,9)$ & $85,2(4,8)$ & $84,6(5,0)$ & 0,515 \\
\hline Geschlecht, weiblich & $115(82,7)$ & $30(83,3)$ & $85(82,5)$ & 1,000 \\
\hline Schulbildungd & & & & 0,319 \\
\hline Abgebrochen & $15(11,5)$ & $6(17,1)$ & $9(9,4)$ & \\
\hline Hauptschule (9 Jahre) & $90(68,7)$ & $26(74,3)$ & $64(66,7)$ & \\
\hline Realschule (10 Jahre) & $17(13,0)$ & $2(5,7)$ & $15(15,6)$ & \\
\hline Gymnasium (13 Jahre) & $9(6,9)$ & $1(2,9)$ & $8(8,3)$ & \\
\hline Familienstand & & & & 0,591 \\
\hline Verheiratet & $22(15,8)$ & $7(19,4)$ & $15(14,6)$ & \\
\hline Verwitwet & $98(70,5)$ & $25(69,4)$ & $73(70,9)$ & \\
\hline Geschieden & $5(3,6)$ & $2(5,6)$ & $3(2,9)$ & \\
\hline Ledig & $14(10,1)$ & $2(5,6)$ & $12(11,7)$ & \\
\hline MMSTe & $15,2(5,3)$ & $14,4(5,2)$ & $15,4(5,4)$ & 0,329 \\
\hline NOSGER & $77,7(17,7)$ & $78,6(17,2)$ & $77,4(17,9)$ & 0,710 \\
\hline Pflegestufeg $^{g}$ & & & & 0,266 \\
\hline Keine & $23(16,5)$ & $3(8,3)$ & $20(19,4)$ & \\
\hline Stufe 1 & $66(47,5)$ & $20(55,6)$ & $46(44,7)$ & \\
\hline Stufe 2 & $50(36,0)$ & $13(36,1)$ & $37(35,9)$ & \\
\hline Psychopharmaka', ja & $77(55,4)$ & $23(63,9)$ & $54(52,4)$ & 0,250 \\
\hline Antidementivai,ja & $29(20,9)$ & $5(13,9)$ & $24(23,3)$ & 0,340 \\
\hline Antidepressivaj, ja & $38(27,3)$ & $10(27,8)$ & $28(27,2)$ & 1,000 \\
\hline Dämpfungsscorek & $-1,4(1,6)$ & $-1,9(1,8)$ & $-1,3(1,4)$ & 0,046 \\
\hline Medikamentenanzahl' & $5,8(2,5)$ & $6,4(2,6)$ & $5,6(2,5)$ & 0,131 \\
\hline $\mathrm{ACB}^{\mathrm{m}}$ & $1,0(1,4)$ & $1,1(1,3)$ & $1,0(1,4)$ & 0,862 \\
\hline $\begin{array}{l}\text { Neurologische sturzprädisponierende Diagno- } \\
\text { sen }^{n} \text {, ja }\end{array}$ & $32(23,0)$ & $9(25,0)$ & $23(22,3)$ & 0,819 \\
\hline \multicolumn{5}{|c|}{$\begin{array}{l}\text { a Sturz mit Verletzung, die die Inanspruchnahme einer ambulanten oder stationären ärztlichen Versorgung der Verletzung zur Folge hatte. b } \\
\text { Mittelwert (Standardabweichung) oder absolute Häufigkeit (\%). c Signifikante p-Werte in Fettdruck (t-Test oder Chi²-Test). d In } 8 \text { Fällen war der } \\
\text { Schulabschluss in den Dokumentationsunterlagen der Einrichtungen nicht enthalten. e,f,g Nähere Beschreibung siehe Abschnitt „Weitere Variablen“. } \\
\text { h Nähere Beschreibung siehe Abschnitt „Pharmakologische Parameter“. ' Es wurden sowohl spezifische Antidementiva (Acetylcholinesterasehemmer } \\
\text { und Memantin) sowie unspezifische Antidementiva der Roten Liste } 2018 \text { eingeschlossen. j Es wurden alle Antidepressiva der Roten Liste } 2018 \\
\text { eingeschlossen. k,l,m Nähere Beschreibung siehe Abschnitt „Pharmakologische Parameter“. " Als neurologische sturzprädisponierende Diagnosen nach } \\
\text { ICD-10 wurden das primäre und sekundäre Parkinson-Syndrom (G20, G21), sonstige extrapyramidale Krankheiten und Bewegungsstörungen (G25), } \\
\text { Epilepsie (G40), Paresen (G80-83) und Hirninfarkte (I63-64) gewertet. Kein Patient der Kohorte litt an Multipler Sklerose (G35). }\end{array}$} \\
\hline
\end{tabular}

von Psychopharmaka sowie das Auftreten von Polypharmazie erfasst. Es wurde die Häufigkeit der Einnahme von dämpfenden Wirkstoffen sowie die Verteilung des „Dämpfungsscores“ untersucht.

Mithilfe einer binär-logistischen Regressionsanalyse wurde der Einfluss des Dämpfungsscores (unabhängige metrische Variable) auf das Auftreten von Sturzereignissen mit Verletzungsfolge ( $j a=1$; nein $=0$ ) (abhängige Variable) im Zeitraum von 12 Monaten nach Studienbeginn untersucht. Als Adjustierungsvariablen wurden Alter, Geschlecht, das Vorliegen einer neurologisch sturzprädisponierenden Diagnose sowie die Zugehörigkeit zur Studiengruppe (Interventions- bzw. Kontrollgruppe) in die Analyse einbezogen. Letztere Variable wurde eingeschlossen, um den Interventionseffekt der psychosozialen MAKS ${ }^{\circledR}$-Therapie zu kontrollieren.

In Sensitivitätsanalysen wurde das Regressionsmodell zusätzlich variiert. Zum einen wurde die Variable „Dämpfungsscore“ durch die Variable „Medikamentenanzahl“ ersetzt. In einer weiteren binär-logistischen Regressionsanalyse wurden beide Variablen ins Modell eingeschlossen. Zusätzlich zu beiden Variablen wurde ein weiteres Modell gerechnet, in dem auch Kognition (MMST), Umfang des Pflegebedarfs (Pflegestufe) sowie die geriatrische Gesamtsymptomatik (NOSGER Summenwert) berücksichtigt wurden. Die Signifikanz potentieller Prädiktoren wurde anhand des WaldKriteriums geprüft. Alle unabhängigen Variablen wurden fest in das Regressionsmodell aufgenommen („Enter-Methode“). Das Signifikanzniveau für sämtliche inferenzstatistischen Berechnungen wurde auf $p=0,05$ festgelegt. Die statistischen Analysen wurden mit SPSS (Version 23 für Windows) durchgeführt.

\section{Ergebnisse}

\section{Stichprobe}

Das Alter der Teilnehmenden variierte zwischen 73 und 98 Jahren. Im Schnitt waren die Demenzerkrankten knapp 85 Jahre alt ( Tab. 1). 82,7\% der Personen waren weiblich. Eine leichte Demenz (MMST-Score von 18-23) lag bei 37,4\% vor, eine mittel- 
schwere Demenz (Score von 10-17) bei 45,3\% und eine schwere Demenz (Score < 10) bei 17,3\%. Die Mehrzahl, knapp $80 \%$, erhielt keine Antidementiva. Im Fall einer Antidementivaverordnung handelte es sich überwiegend ( $90 \%$ ) um einen spezifischen Wirkstoff (Acetylcholinesterasehemmer oder Memantin). Knapp ein Viertel (23,0\%) der Demenzerkrankten litt an einer sturzprädisponierenden neurologischen Erkrankung, wie z. B. dem Parkinson-Syndrom oder Epilepsie ( $>$ Tab. 1). Im untersuchten Zeitraum von 12 Monaten verstarben 24 Personen (17,3\%). Diese Zahl resultiert aus 14 primären Todesfällen ( $\triangleright$ Abb. 1) sowie weiteren 10 Todesfällen, die nach einem anderen Dropoutgrund eingetreten waren (z. B. nach Eintritt von Bettlägerigkeit oder nach längerem Krankenhausaufenthalt).

\section{Sturzereignisse mit Verletzungsfolge}

Im gesamten Beobachtungszeitraum kam es bei 36 der 139 eingeschlossenen Personen zu Sturzereignissen mit Verletzungsfolge. Ein Drittel der Betroffenen (12 Personen) stürzte mehrfach: 9 Personen 2-mal, eine Person 3-mal und 2 Personen 4-mal. Insgesamt wurden im Beobachtungszeitraum von 12 Monaten 53 Stürze registriert. $13 \%$ der Stürze mit Verletzungsfolge wurden stationär behandelt, $87 \%$ wurden ambulant behandelt.

\section{Medikamentenanzahl}

Im Schnitt erhielten die Pflegeheimbewohnerinnen und -bewohner 5,8 verschiedene systemisch wirkende Arzneimittel. Eine Person $(0,7 \%)$ erhielt keine Medikation. Ein Drittel $(33,1 \%)$ der Demenzkranken erhielt 1 bis 4 Arzneimittel, über die Hälfte (58,3\%) nahm 5 bis 9 Medikamente ein und 11 Personen (7,9\%) erhielten zehn oder mehr verschiedene Arzneimittel. Polypharmazie lag also bei $66,2 \%$ der demenzkranken Pflegeheimbewohnerinnen und -bewohner vor.

\section{PRISCUS-Liste}

Bei knapp $30 \%$ der untersuchten Heimbewohnerinnen und -bewohner (41 Personen) wurde mindestens ein Wirkstoff der PRISCUS-Liste verschrieben. Drei Personen erhielten 2 Wirkstoffe. Die am häufigsten verordneten Wirkstoffe der PRISCUS-Liste waren Zopiclon und Digoxin. Beide Wirkstoffe wurden 4-mal verordnet. Danach folgten Doxepin, Amitriptylin und Haloperidol mit jeweils 3 Verordnungen.

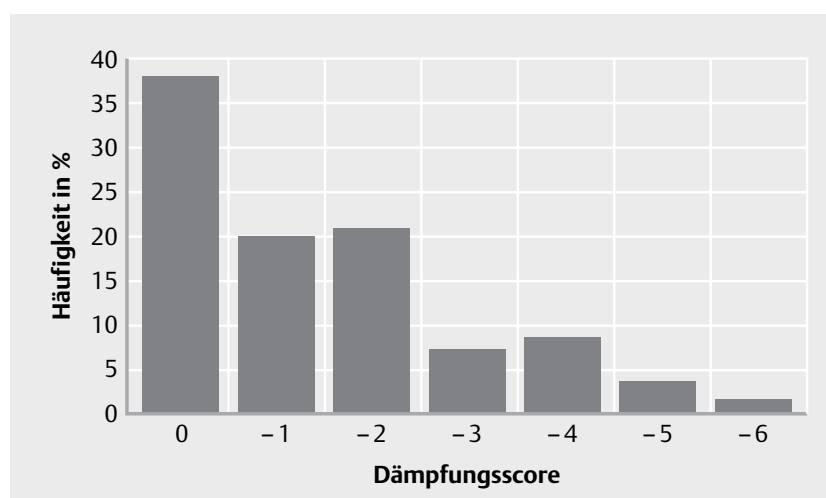

Abb. 2 Verteilung des Dämpfungsscores in der Stichprobe.

\section{ACB-Liste}

Die Hälfte der Heimbewohnerinnen und -bewohner (50,4\%) erhielt mindestens einen Wirkstoff mit anticholinerger Wirkung. Ein summativer ACB-Score von 1 lag bei 25,9\% (36 Personen) vor. 5,8\% (8 Personen) der Stichprobe hatten einen ACB-Score von 2. Bei 18 Personen (12,9\%) wurde ein Score von 3 erreicht. Sechs Personen $(4,3 \%)$ wiesen einen ACB-Score von 4 auf, je eine Person ( $0,7 \%$ ) erreichte einen $A C B-S c o r e$ von 5 bzw. 7. Der am häufigsten verordnete Wirkstoff der ACB-Liste war Metoprolol mit 19 Verordnungen. Danach folgten Risperidon (16 Personen), Furosemid (14 Personen), Quetiapin (6 Personen) und Digoxin (4 Personen).

\section{Psychopharmaka}

Über die Hälfte der demenzerkrankten Personen (55,4\%) erhielt mindestens ein Psychopharmakon. Davon erhielten 67,5\% (52 Personen) ein Psychopharmakon, 24,7\% (19 Personen) 2 Psychopharmaka, 6,5\% (5 Personen) drei und 1,3\% (1 Person) vier Psychopharmaka.

\section{Dämpfungsscore}

Mehr als die Hälfte der Pflegeheimbewohnerinnen und -bewohner $(61,9 \%)$ erhielt zentralnervös dämpfende Arzneimittel. Von dieser Personengruppe enthielt der Dämpfungsscore bei 38 Personen (44,2\%) ein stark dämpfendes Arzneimittel. Bei weiteren 16 Personen (18,6\%) flossen in den Score 2 stark dämpfende Wirkstoffe ein. Eine Person erhielt drei stark dämpfende Arzneimittel. Die Verteilung des Dämpfungsscores ist in $\mathbf{A} \mathbf{A b} \mathbf{b} . \mathbf{2}$ dargestellt. Bei keiner Person lag ein positiver Wert des Dämpfungsscores, also im zentralnervös aktivierenden Bereich, vor. Die häufigste als zentralnervös dämpfend eingestufte Medikation war Melperon (20 Personen), gefolgt von Risperidon (16 Personen), Memantin (15 Personen), Mirtazapin (9 Personen) und Pipamperon (7 Personen).

Bivariat betrachtet zeigte sich nur für die Variable Dämpfungsscore ( $\triangleright$ Tab. 1) ein statistisch signifikanter Zusammenhang mit der Zielgröße „Sturz mit Verletzungsfolge“. Daher wurde in einer binär-logistischen Regression der Dämpfungsscore altersund geschlechtsadjustiert auf seinen Einfluss auf die Zielgröße untersucht. Ein potentieller Effekt der psychosozialen Intervention (MAKS ${ }^{\circledR}$ ja oder nein) sowie Vorhandensein einer sturzprädisponierenden Diagnose (ja oder nein) wurde hierbei ebenfalls kontrolliert. Auch im multivariaten Zusammenhang erwies sich nur der Dämpfungsscore als signifikanter Prädiktor $(p=0,045)$ für das Auftreten eines schwerwiegenden Sturzereignisses ( $>$ Tab. 2). Je ausgeprägter die aufsummierte zentralnervöse Dämpfung der verordneten Arzneimittel zum Zeitpunkt des Studienbeginns war, das heißt je niedriger der Summenwert (negative Werte!), desto wahrscheinlicher ereignete sich innerhalb von 12 Monaten nach Studienbeginn ein Sturz mit Verletzungsfolge, der ärztlich behandelt werden musste.

\section{Sensitivitätsanalysen}

Die Medikamentenanzahl, die ungewichtete Anzahl aller Dauermedikamente, enthält neben allen anderen Arzneimitteln auch die zentral dämpfend wirkenden. Deshalb korrelieren beide Variablen signifikant $(p<0,001)$ mit $r=-0,33$ (Pearson). Die Medikamentenanzahl ist zwar bei denjenigen mit Sturzereignis größer als bei den- 
> Tab. 2 Multivariate Analyse der Assoziation des Dämpfungsscores auf das Ereignis „Sturz mit Verletzungsfolge innerhalb von 12 Monaten“ ( 0 =nein, 1 =ja).

\begin{tabular}{|c|c|c|c|c|}
\hline Unabhängige Variablen & Regressionskoeffizient & Wald-Koeffizient & $p$-Werta & Odds ratio (95\% Konfidenzintervall) \\
\hline Dämpfungsscore & $-0,25$ & 4,00 & 0,045 & $0,78(0,61-1,00)$ \\
\hline Zugehörigkeit zur Studiengruppe & $-0,48$ & 1,43 & 0,232 & $0,62(0,28-1,36)$ \\
\hline Alter & 0,03 & 0,55 & 0,457 & $1,03(0,95-1,11)$ \\
\hline Geschlecht & $-0,04$ & 0,01 & 0,943 & $0,96(0,34-2,73)$ \\
\hline Neurologische sturzprädisponierende Diagnosen & 0,20 & 0,18 & 0,670 & $1,22(0,49-3,07)$ \\
\hline
\end{tabular}

- Tab. 3 Sensitivitätsanalyse mit Einschluss von Medikamentenanzahl, Kognition (MMST), Umfang des Pflegebedarfs (Pflegestufe) und geriatrischer Gesamtsymptomatik (NOSGER Summenwert).

\begin{tabular}{|l|l|l|l|l|}
\hline Unabhängige Variablen & Regressionskoeffizient & Wald-Koeffizient & $\boldsymbol{p}$-Wert & Odds ratio (95\% Konfidenzintervall) \\
\hline Dämpfungsscore & $-0,19$ & 2,02 & 0,156 & $0,83(0,63-1,08)$ \\
\hline Zugehörigkeit zur Studiengruppe & $-0,51$ & 1,54 & 0,215 & $0,60(0,27-1,34)$ \\
\hline Alter & 0,03 & 0,41 & 0,524 & $1,03(0,95-1,11)$ \\
\hline Geschlecht & $-0,08$ & 0,02 & 0,881 & $0,92(0,32-2,64)$ \\
\hline Neurologische sturzprädisponierende Diagnosen & 0,16 & 0,10 & 0,747 & $1,17(0,46-2,99)$ \\
\hline Mini-Mental-Status-Test & 0,00 & 0,63 & 0,426 & $1,00(1,00-1,00)$ \\
\hline Medikamentenanzahl & 0,09 & 0,99 & 0,319 & $1,09(0,92-1,30)$ \\
\hline Pflegestufe, dichotom & $-0,06$ & 0,02 & 0,892 & $0,94(0,39-2,26)$ \\
\hline NOSGER Summenwert & 0,00 & 0,02 & 0,889 & $1,00(0,98-1,03)$ \\
\hline
\end{tabular}

jenigen ohne Sturz (6,4 vs. 5,6 Arzneimittel), der Unterschied ist jedoch nicht signifikant ( $p=0,131$, vgl. $\triangleright$ Tab. 1$)$.

Ersetzt man die Variable „Dämpfungsscore“ in der Regression durch die Variable „Medikamentenanzahl“, resultiert ein Wald-Koeffizient von 2,51 ( $p=0,113$ ) für die Anzahl der Arzneimittel. Die Medikamentenanzahl weist also einen geringeren und nicht signifikanten multivariaten Zusammenhang mit der Zielgröße im Vergleich zum Dämpfungsscore auf.

Werden beide Variablen in die Regression aufgenommen, resultiert für den Dämpfungsscore zwar ein größerer Wald-Koeffizient $(2,40)$ als für die Medikamentenanzahl $(0,99)$, der prädiktive Effekt beider Variablen ist jedoch nicht signifikant ( $p=0,121$ bzw. $p=0,321$ ). Nimmt man weitere Variablen in die Regressionsanalyse auf - Kognition (MMST-Wert), Umfang des Pflegebedarfs (Pflegestufe dichotom) und geriatrische Gesamtsymptomatik (NOSGER Summenwert) - ändert sich an den Wald-Koeffizienten für Dämpfungsscore und Medikamentenanzahl wenig ( $>$ Tab. 3). Die neu aufgenommenen Variablen besitzen keinen relevanten bzw. signifikanten Vorhersagewert für die Zielgröße „Sturz mit Verletzungsfolge ja/nein“.

\section{Diskussion}

Die Ergebnisse zeigen eine signifikante Assoziation zwischen der Einnahme von zentralnervös dämpfenden Arzneimitteln und Stürzen mit ärztlich behandlungsbedürftiger Verletzungsfolge bei Demenzerkrankten innerhalb des folgenden Jahres. Eine stärkere psychomotorische Dämpfung führte zu vermehrten Sturzereignissen mit Verletzungsfolge. Die Substanzgruppen Sedativa, Antidepressiva und Antipsychotika enthalten besonders häufig als stark dämpfend eingestufte Arzneimittel. Die Zahl aller als Dauermedikation eingenommener Arzneimittel ist kein so starker Prädiktor für Stürze mit ärztlich behandlungsbedürftiger Verletzungsfolge wie der „Dämpfungsscore“.

In mehreren Studien wurde gezeigt, dass ältere Menschen mit kognitiven Defiziten wie Demenz häufiger stürzen als gleichaltrige ohne einschlägige Diagnose [3,4]. Laut der für die deutschsprachige Bevölkerung repräsentativen Studie "Gesundheit in Deutschland aktuell 2010«(GEDA 2010), die vom Robert Koch Institut veröffentlicht wurde und bei der über 22000 Personen in Deutschland befragt wurden, endet fast jeder zweite Sturz (47,2\%) bei über 60-Jährigen mit einer Fraktur [21]. Die Kosten, die in Deutschland allein für die medizinische Behandlung von Oberschenkel- und Hüftverletzungen anfallen, beliefen sich im Jahr 2015 nach Angaben des Statistischen Bundesamtes auf über 3 Milliarden Euro [2]. Neben den körperlichen Folgen eines Sturzes kommt es bei vielen Personen nach einem Sturzereignis zu einer Sturzangst, die wiederum zu verringerter Aktivität im Alltag führt und oft soziale Isolation und eine Verringerung der Lebensqualität bedeutet [22].

Der hier vorgestellte Dämpfungsscore greift somit eine besonders für Demenzerkrankte relevante Wirkung, die zentralnervöse Dämpfung, auf, die in den bisher verwendeten Kategorisierungen von Arzneimitteln nicht explizit zur Geltung kommt. Das betrifft die Kategorien Psychopharmaka, „Anticholinergic Cognitive Burden" und Polypharmazie.

Die Einbeziehung der Variable „Sturzprädisponierende neurologische Erkrankung“ zeigte keinen eigenständigen signifikanten Erklärungsanteil auf Sturz mit behandlungsbedürftiger Verletzungsfolge. Eine mögliche Erklärung hierfür ist, dass alle Vergleichspersonen an Demenz erkrankt waren und somit bereits bei allen Personen durch die Erkrankung ein erhöhtes Sturzrisiko vorlag, was den relativen Einfluss einer weiteren sturzprädisponierenden Er- 
krankung verringert. Ein weiterer Grund könnte sein, dass schwere Formen sturzprädisponierender neurologischer Erkrankungen durch das Ausschlusskriterium „Pflegestufe 3“ nicht in die Studie eingeschlossen worden waren. Da in unserer Studie nur Stürze mit ärztlich behandlungsbedürftigen Folgen erfasst wurden, kann nicht ausgeschlossen werden, dass es bei Personen mit sturzprädisponierenden neurologischen Erkrankungen häufiger zu Sturzereignissen ohne Verletzungsfolge kam.

In der Untersuchung von Wucherer et al. [23], die 448 Demenzerkrankte in Mecklenburg-Vorpommern einschloss, die im häuslichen Umfeld wohnten, erhielten 22,1\% der Personen mindestens einen Wirkstoff der PRISCUS-Liste. Für hospitalisierte geriatrische Patientinnen und Patienten in Bayern, die auch aber nicht nur demenzerkrankte ältere Menschen beinhalten, betrug dieser Wert 25,9\% [24]. In unserer Stichprobe erhielten knapp 30\% der Pflegeheimbewohnerinnen und -bewohner solche Wirkstoffe. Unter Berücksichtigung der unterschiedlichen Settings beziehungsweise der unterschiedlichen Stichprobenzusammensetzung kann von einer vergleichbaren Größenordnung ausgegangen werden.

In der retrospektiven Kohortenstudie von Pfistermeister et al. [18], die knapp 90000 geriatrische, hospitalisierte Patientinnen und Patienten aus der „Geriatrie in Bayern-Datenbank“ (GiB-DAT) einschloss, erhielten 46,3\% der Personen mindestens einen Wirkstoff der ACB-Liste. Mit 50,4\% erhalten die Demenzerkrankten unserer Studie vergleichbar viele anticholinerg wirkende Arzneimittel. Angesichts des Risikos einer kognitiven Verschlechterung bei der Gabe dieser Arzneimittel bei Betroffenen mit Alzheimer Demenz, der häufigsten degenerativen Demenzform, wäre es wünschenswert, diesen Wert zu senken. Diese Hypothese müsste wissenschaftlich durch große prospektive randomisierte Studien noch näher untersucht werden.

Antipsychotika sind ein kritisch zu betrachtender Anteil der Psychopharmaka bei Demenz. Der Einsatz von Antipsychotika bei Demenzerkrankten ist laut aktueller S3-Leitlinie für Demenzen wahrscheinlich mit einem erhöhten Risiko für Mortalität und für zerebrovaskuläre Ereignisse assoziiert [25]. Außerdem ist mit einem erhöhten Auftreten von motorischen Nebenwirkungen (Parkinsonoid) zu rechnen. In der Studie von Bohlken et al. [26] wurden Daten von knapp 15000 Demenzerkrankten in deutschen neurologischen und psychiatrischen Praxen mit einem Alter von über 60 Jahren in die Studie eingeschlossen. 47,7\% der untersuchten Personen erhielten 2 Jahre nach ihrer Demenzdiagnose Antipsychotika. Bei Huber et al. [27] wurde die Medikation von 8000 Demenzerkrankten in Pflegeheimen in Deutschland retrospektiv für das Jahr 2008 untersucht. 51,6\% der Personen erhielten eine antipsychotische Medikation. Die Gesamtrate des Einsatzes von Psychopharmaka (einschließlich Antipsychotika) beträgt in unserer Studie $55,4 \%$ und hat somit einen vergleichbar hohen Wert. Angesichts der eindeutig vor erheblichen unerwünschten Wirkungen warnenden Aussage der S3-Leitlinie Demenzen ist darauf hinzuwirken, dass dieser hohe Wert reduziert werden sollte und zwar durch den alternativen Einsatz wirksamer, die neuropsychiatrischen Symptome günstig beeinflussender, sogenannter „psychosozialer“ Interventionen, wie etwa der multimodalen, nichtpharmakologischen MAKS $^{\circledR}$-Therapie [12, 28].

Der Versorgungsreport des Wissenschaftlichen Instituts der AOK (WIdO) enthält Daten von über 65-jährigen, gesetzlich versi- cherten Personen. Bei $42 \%$ dieser Personen wurde Polypharmazie festgestellt [29]. In unserer Studie erhielten sogar zwei Drittel $(66,2 \%)$ der Demenzerkrankten mindestens fünf verschiedene Medikamente.

Als Stärke der Studie ist hervorzuheben, dass mit dieser Arbeit zum ersten Mal eine Kombination aus der Assoziation eines summativen Wertes aller relevanten zentral dämpfenden Arzneimittel mit einem „harten“, patienten- und gesundheitsökonomisch relevanten Outcome (Sturz mit ärztlich behandlungsbedürftiger Verletzungsfolge) untersucht wird. Dieses Outcome wurde über einen Zeitraum von 12 Monaten lückenlos dokumentiert und es wurden außerdem sämtliche Komorbiditäten erfasst.

Als Limitationen sind zu nennen, dass aufgrund der Verwendung der Ausgangsdaten einer Interventionsstudie nur eine begrenzte Teilnehmerzahl für die vorliegende Analyse verwendet werden konnte. Alkoholkonsum als weiterer Risikofaktor für Sturzereignisse wurde im Rahmen der Studie nicht erfasst und konnte deshalb nicht in die Analyse einbezogen werden. Einschränkend ist festzustellen, dass in den Dämpfungsscore die Bewertung der Dosierung (adäquat versus inadäquat) nicht einbezogen werden konnte, da diese Information für die Auswertung nicht zur Verfügung stand. Außerdem erfolgte keine Prüfung auf individuelle pharmakokinetische und pharmakodynamische Besonderheiten z. B. durch Plasmakonzentrationskontrollen oder durch Berücksichtigung von Arzneimittelinteraktionen. Die Auswertung erfolgte anhand der Eingangsmedikation, Veränderungen der Medikation im weiteren Verlauf wurden nicht erfasst. Weiterhin wurde ein additiver Effekt mehrerer zentralnervös dämpfender Arzneimittel angenommen. Diese Annahme ist in zukünftigen Studien zu überprüfen. Wünschenswert ist deshalb eine Weiterentwicklung und Präzisierung des Dämpfungsscores, um damit Auswirkungen der Arzneimitteltherapie bei Demenz noch genauer untersuchen zu können. Die Studienpopulation bezieht sich auf Pflegeheimbewohnerinnen und -bewohner, die an einer Demenz leiden. In weiteren Studien zum Dämpfungsscore sollten auch ältere, zuhause lebende Menschen ohne Demenz einbezogen werden.

\section{FAZIT FÜR DIE PRAXIS}

Anzustreben ist ein pharmakologisches Therapieregime bei Demenz, das möglichst wenig zentral dämpfende Arzneimittel einsetzt bzw. bei Wahlmöglichkeit die Entscheidung für weniger zentral dämpfende Arzneimittel präferiert. Eine Arzneimitteltherapie bei Demenz sollte außerdem darauf ausgerichtet sein, Arzneimittel mit anticholinergen oder mortalitätserhöhenden unerwünschten Wirkungen nach Möglichkeit zu vermeiden.

\section{Danksagung}

Besonderer Dank gilt allen Studienteilnehmerinnen und -teilnehmern der MAKS(R)-Studie. Die vorliegende Arbeit wurde von Theresa Lippert als Teil der Anforderungen zur Erlangung des Grades “Dr. med." an der Medizinischen Fakultät der Friedrich-AlexanderUniversität Erlangen-Nürnberg (FAU) angefertigt. 


\section{Interessenkonflikt}

Die Autorinnen/Autoren geben an, dass kein Interessenkonflikt besteht.

\section{Literatur}

[1] Statistisches Bundesamt. Gesundheit Krankheitskosten 2002, 2004, 2006 und 2008. Ausgabe 2015. Im Internet: www.destatis.de; Stand: 18.04.2019

[2] Statistisches Bundesamt. Gesundheit Krankheitskosten 2015. Im Internet: www.destatis.de; Stand: 18.04.2019

[3] Harlein J, Dassen T, Halfens RJ et al. Fall risk factors in older people with dementia or cognitive impairment: a systematic review. J Adv Nurs 2009; 65: 922-933

[4] Muir SW, Gopaul K, Montero Odasso MM. The role of cognitive impairment in fall risk among older adults: a systematic review and meta-analysis. Age Ageing 2012; 41: 299-308

[5] Galik E, Holmes S, Resnick B. Differences between moderate to severely cognitively impaired fallers versus nonfallers in nursing homes. Am J Alzheimers Dis Other Demen 2018; 33: 247-252

[6] Ray WA, Thapa PB, Gideon P. Benzodiazepines and the risk of falls in nursing home residents. J Am Geriatr Soc 2000; 48: 682-685

[7] Leipzig RM, Cumming RG, Tinetti ME. Drugs and falls in older people: a systematic review and meta-analysis: I. Psychotropic drugs. J Am Geriatr Soc 1999; 47: 30-39

[8] Thapa PB, Gideon P, Cost TW et al. Antidepressants and the risk of falls among nursing home residents. N Engl J Med 1998; 339: 875-882

[9] Perttila NM, Ohman H, Strandberg TE et al. How do community-dwelling persons with Alzheimer disease fall? Falls in the FINALEX study. Dement Geriatr Cogn Dis Extra 2017; 7: 195-203

[10] Rapp K, Buchele G, Dreinhofer K et al. Epidemiology of hip fractures: Systematic literature review of German data and an overview of the international literature. Z Gerontol Geriatr 2019; 52: 10-16

[11] Luttenberger K, Hofner B, Graessel E. Are the effects of a non-drug multimodal activation therapy of dementia sustainable? Follow-up study 10 months after completion of a randomised controlled trial. BMC Neurol 2012; 12: 151

[12] Luttenberger K, Donath C, Uter W et al. Effects of multimodal nondrug therapy on dementia symptoms and need for care in nursing home residents with degenerative dementia: a randomized-controlled study with 6-month follow-up. J Am Geriatr Soc 2012; 60: 830-840

[13] Graessel E, Stemmer R, Eichenseer B et al. Non-pharmacological, multicomponent group therapy in patients with degenerative dementia: a 12-month randomizied, controlled trial. BMC Med 2011; 9: 129

[14] Folstein MF, Folstein SE, McHugh PR. "Mini-mental state". A practical method for grading the cognitive state of patients for the clinician. J Psychiatr Res 1975; 12: 189-198
[15] Hovstadius B, Hovstadius K, Astrand B et al. Increasing polypharmacy - an individual-based study of the Swedish population 2005-2008. BMC Clin Pharmacol 2010; 10: 16

[16] Holt S, Schmiedl S, Thürmann PA. Potentially inappropriate medications in the elderly: the PRISCUS list. Dtsch Ärztebl Int 2010; 107: 543-551

[17] Aging Brain Care. Anticholinergic Cognitive Burden Scale 2012 Update. Im Internet: www.miltonkeynesccg.nhs.uk/resources/uploads/ACB_ scale_-_legal_size.pdf Stand: 18.04.2019

[18] Pfistermeister B, Tümena T, Gassmann KG et al. Anticholinergic burden and cognitive function in a large German cohort of hospitalized geriatric patients. PLoS One 2017; 12: e0171353

[19] S3-Leitlinie Behandlung von Angststörungen. Im Internet: https:// www.awmf.org/leitlinien.html Stand: 18.04.2019

[20] Spiegel R, Brunner C, Ermini-Fünfschilling D et al. A new behavioral assessment scale for geriatric out- and in-patients: the NOSGER (Nurses' Observation Scale for Geriatric Patients). Am J Geriatr Psychiatry 1991; 39: 339-347

[21] Gesundheitsberichterstattung des Bundes RKI. Das Unfallgeschehen bei Erwachsenen in Deutschland. Ergebnisse des Unfallmoduls der Befragung "Gesundheit in Deutschland aktuell 2010«. Im Internet: http://www.rki.de/DE/Content/Gesundheitsmonitoring/Gesundheitsberichterstattung/GBEDownloadsB/unfallbericht_geda.pdf Stand: 18.04.2019

[22] Visschedijk J, Achterberg W, Van Balen R et al. Fear of falling after hip fracture: a systematic review of measurement instruments, prevalence, interventions, and related factors. J Am Geriatr Soc 2010; 58 : 1739-1748

[23] Wucherer D, Eichler T, Hertel J et al. Potentially inappropriate medication in community-dwelling primary care patients who were screened positive for dementia. J Alzheimers Dis 2017; 55: 691-701

[24] Fromm MF, Maas R, Tümena T et al. Potentially inappropriate medications in a large cohort of patients in geriatric units: association with clinical and functional characteristics. Eur J Clin Pharmacol 2013; 69: 975-984

[25] DGPPN, DGN. S3-Leitlinie „Demenzen“ (Langversion Januar 2016). Im Internet: https://www.awmf.org/leitlinien.html Stand: 18.04.2019

[26] Bohlken J, Booker A, Kostev K. High prevalence of antipsychotic medication use in dementia patients in German neuropsychiatric practices. Fortschr Neurol Psychiatr 2017; 85: 345-351

[27] Huber M, Kolzsch M, Rapp MA et al. Antipsychotic drugs predominate in pharmacotherapy of nursing home residents with dementia. Pharmacopsychiatry 2012; 45: 182-188

[28] Straubmeier M, Behrndt EM, Seidl $\mathrm{H}$ et al. Non-pharmacological treatment in people with cognitive impairment. Dtsch Ärztebl Int 2017; 114: 815-821

[29] Mosshammer D, Haumann H, Morike K et al. Polypharmacy - an upward trend with unpredictable effects. Dtsch Ärztebl Int 2016; 113: 627-633 